\title{
HIV infection newly diagnosed in Northern Italy; evolving trends
}

\author{
Roberto Manfredi \\ From $16^{\text {th }}$ International Symposium on HIV and Emerging Infectious Diseases \\ Marseille, France. 24-26 March 2010
}

\section{Background}

To assess prospectively all newly diagnosed cases of HIV infection performed at our reference centre, which serves around 800,000 inhabitants of the Bologna metropolitan area.

\section{Methods}

All patients with a newly diagnosed HIV infection were initially assessed according to several demographic, epidemiological, diagnostic, clinical, and laboratory features.

\section{Results}

From June 2006 up to December 2008 (31 months), 162 patients were first diagnosed with HIV disease (mean 5.2 novel cases per month), and 78 of them were judged to have a recent infection (as established on the ground of a specific "avidity" serologic testing). Males greatly prevailed over females (93 versus 69 cases), while homosexual exposure (53 cases) was prevalent over heterosexual one (38 patients), and only two novel cases were registered among i.v. drug users. The median age at diagnosis was 36.2 years, while the main laboratory parameters showed a mean CD4+ count of 502 cells $/ \mu \mathrm{L}$, and a mean HIV-RNA load of $8.21 \times 10^{4}$ copies $/ \mathrm{mL}$. Although subtype B of HIV greatly prevailed (141 cases: 87\%), also subtypes A-A1, and recombinant HIV virions were found among newly infected patients. When conducting a genotypic resistance assay including all available antiretroviral agents, the overall prevalence of primary mutations accounted for $13 \%$ of newly infected patients: $11.1 \%$ of them had one or more mutations of the reverse transcriptase gene, and $9.3 \%$ of subjects had one or more mutations of the protease inhibitor gene (including one case of multiple mutations, probably conferring resistance extended to the third-generation protease inhibitor tipranavir). The majority of patients with recent infection (50 out of 78: 64.1\%) were asymptomatic or paucisymptomatic, while a full-blown AIDS or a symptomatic disease were present in 18 and 10 cases respectively.

\section{Discussion}

Nothwistanding the massive prevention campaigns of the last two decades, HIV infection continues to spread predominantly via sexual route, and may increasingly involve immigrants. A delayed-missed recognition of HIV infection poses patients at a very high risk to develop HIV-related disorders (since these subjects could not take advantage from antiretroviral therapy). Non-subtype B viruses, recombinant viruses, and HIV strains already encoding for resistance against different antiretroviral compounds are of significant concern. A permanent, active monitoring of this phenomenon and its correlates is strongly needed.

Published: 11 May 2010

doi:10.1186/1742-4690-7-S1-P102

Cite this article as: Manfredi: HIV infection newly diagnosed in Northern Italy; evolving trends. Retrovirology 2010 7(Suppl 1):P102.

Correspondence: Roberto.manfredi@unibo.it

Infectious Diseases, University of Bologna, S. Orsola Hospital, Bologna, Italy 